\title{
A phase I study of the tyrosine kinase inhibitor anlotinib combined with platinum/pemetrexed-based chemotherapy in untreated nonsquamous non-small-cell lung cancer
}

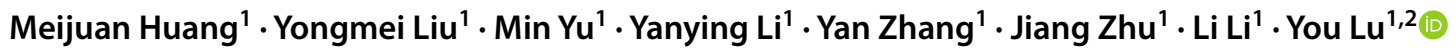

Received: 7 July 2021 / Accepted: 12 September 2021 / Published online: 1 November 2021

(c) The Author(s) 2021

\begin{abstract}
Summary
Background. Anlotinib hydrochloride is an oral small molecule inhibitor of multiple tyrosine kinases, and it has been approved as a third-line therapy for patients with advanced non-small-cell lung cancer (NSCLC) in China. This dose-exploration study was designed to investigate the feasibility of anlotinib in combination with other chemotherapy regimens in patients with nonsquamous NSCLC. Methods. This phase I study followed a $3+3$ dose reduction design with three doses of anlotinib (12 mg, $10 \mathrm{mg}$, and $8 \mathrm{mg})$. Anlotinib was given at an initial dose of $12 \mathrm{mg}$ with pemetrexed $\left(500 \mathrm{mg} / \mathrm{m}^{2}\right)$ plus cisplatin $\left(75 \mathrm{mg} / \mathrm{m}^{2}\right)$ or carboplatin $(\mathrm{AUC}=5)$ on 21-day cycles for 4 cycles. The primary goal of the study was to identify the maximum tolerated dose (MTD), and secondary endpoints included progression-free survival (PFS) and overall survival (OS). Results. A total of eight participants were enrolled. Dose-limiting toxicities (DLTs) were observed in two patients (pts) at anlotinib $12 \mathrm{mg}$ (grade 3 hand-foot syndrome and grade 3 appetite loss). No DLTs occurred with $10 \mathrm{mg}$ anlotinib, and the MTD was $10 \mathrm{mg}$. Among seven evaluable pts, four achieved a confirmed partial response (PR), and three had stable disease (SD). With a median follow-up of 10.05 months, the median PFS was 7.00 months (95\% CI: 2.76 to NE). Grade 3 treatment-related adverse events (TRAEs) included appetite loss $(n=2)$, hypertension $(n=2)$, thrombocytopenia $(n=1)$, diarrhea $(n=1)$ and hand-foot syndrome $(n=1)$. No grade 4 or grade 5 TRAEs were observed during the treatment. Conclusion. The feasible dose of anlotinib in combination with platinum/pemetrexed-based chemotherapy as a first-line regimen was $10 \mathrm{mg}$, which was well tolerated and showed promising antitumor activity in advanced nonsquamous NSCLC.
\end{abstract}

Keywords Anlotinib $\cdot$ Phase I study $\cdot$ Non-small-cell lung cancer $\cdot$ first-line therapy

\section{Introduction}

In recent years, various antiangiogenic therapies have been evaluated in combination with platinum-based chemotherapy as a first-line treatment for advanced NSCLC without driver gene mutations [1, 2]. However, only bevacizumab, a humanized monoclonal antibody that binds vascular endothelial growth factor (VEGF), has been approved in the clinic [3-5]. Small molecule tyrosine kinase inhibitors (TKIs) with antiangiogenic activity, such as sorafenib, cediranib and vandetanib, have also been investigated in

You Lu

radyoulu@hotmail.com

1 Department of Thoracic Oncology, Cancer Center and State Key Laboratory of Biotherapy, West China Hospital, Sichuan University, Chengdu 610041, China

2 Laboratory of Clinical Cell Therapy, West China Hospital, Sichuan University, Chengdu 610041, China this setting, but no significant improvement in survival was observed. In addition, most of these oral TKIs result in added toxicities. Therefore, clinical trials involving antiangiogenic TKIs in combination with chemotherapy for untreated NSCLC are worthy of further exploration.

Anlotinib is an oral angiokinase inhibitor that blocks VEGFR 1 to 3, platelet-derived growth factor receptor (PDGFR) $\alpha$ and $\beta$, and fibroblast growth factor receptor (FGFR) 1 to $4[6,7]$. In addition, the receptor kinases RET, c-Kit and c-Met are also inhibited. With a low half maximal inhibitory concentration $\left(\mathrm{IC}_{50}\right)$, anlotinib has shown promising antitumor activity in various tumors, including NSCLC, in previous studies $[6,8]$, and it is the only CFDA-approved angiokinase inhibitor for advanced NSCLC [9]. Based on the pharmacokinetics of anlotinib, limited drug-drug interactions allow its combination with cytotoxic drugs. Therefore, an anlotinib-platinum-pemetrexed (APP) regimen was investigated in this trial. Considering the toxicity profile of 
anlotinib monotherapy as well as the predicted intolerance of the combination therapy, a dose-reduction design was employed, reducing the administered dose of anlotinib from 12 to $10 \mathrm{mg}$ and $8 \mathrm{mg}$.

\section{Patients and methods}

\section{Study population}

Eligible patients had histologically or cytologically confirmed stage IIIB/IIIC/IV nonsquamous NSCLC; were negative for mutations of EGFR\ALKIROS1; were aged 18 to 70; never received any systematic treatment (including immunotherapy); had an Eastern Cooperative Oncology Group (ECOG) performance status 0-1; had an expected survival time $\geq 3$ months; and presented no major organ dysfunction. Patients were excluded if they had active brain metastases, uncontrolled hypertension, severe cardiovascular diseases, or coagulation abnormalities.

This study was reviewed and approved by the Institutional Review Board of West China Hospital, Sichuan University. Written informed consent was obtained from all participants. This study is registered with ClinicalTrials.gov, number NCT04012619.

\section{Study design}

This study employed a standard $3+3$ dose reduction design, and eligible patients received an anlotinib chemotherapy regimen after a 21-day cycle for 4 cycles. According to the ALTER0303 study, the initial dose of anlotinib was set as $12 \mathrm{mg} /$ day with a 2-week on/1-week off schedule. The dose was reduced to $10 \mathrm{mg} /$ day and $8 \mathrm{mg} /$ day in sequence depending on observed DLTs in cycle 1 . If there were no DLTs, the dose of anlotinib in the combined chemotherapy regimen was determined to be $12 \mathrm{mg}$ /day. If a DLT occurred in $\geq 2$ of 3 enrolled subjects, the initial dose was reduced to $10 \mathrm{mg} /$ day. If DLT occurred in 1 of 3 subjects, the dose level was followed up, and 3 additional subjects were enrolled. If 1 DLT occurred in the last 3 subjects, the dose was reduced to $10 \mathrm{mg} /$ day. Pemetrexed $\left(500 \mathrm{mg} / \mathrm{m}^{2}\right)$ and either cisplatin $\left(75 \mathrm{mg} / \mathrm{m}^{2}\right)$ or carboplatin (AUC $\left.=5\right)$ were intravenously given on Day 1 of each cycle. Patients who had disease control after the combination regimen continued to receive anlotinib maintenance until disease progression was observed.

\section{Assessments}

Adverse events (AEs) were monitored during the study and summarized according to the National Cancer Institute Common Terminology Criteria for Adverse Events (NCI CTCAE) version 4.0. Based on Response Evaluation Criteria In Solid Tumors (RECIST) version 1.1, the tumor
Table 1 Patient characteristics at baseline

\begin{tabular}{ll}
\hline Characteristics & Anlotinib $(\mathrm{n}=8)$ \\
\hline Age, median (range), years & $62.0(39.0-69.0)$ \\
Gender, n (\%) & \\
Male & $5(62.5)$ \\
Female & $3(37.5)$ \\
ECOG, n (\%) & \\
0 & $1(12.5)$ \\
1 & $7(87.5)$ \\
Stage, n (\%) & \\
IVA & $5(62.5)$ \\
IVB & $3(37.5)$ \\
Smoking history, n (\%) & \\
Ever & $4(50.0)$ \\
Never & $4(50.0)$ \\
\hline
\end{tabular}

response was evaluated by computerized tomography (CT) or magnetic resonance imaging (MRI) at baseline and subsequently every 6 weeks until study termination. Patients who achieved a complete response (CR) or partial response (PR) were required to have efficacy confirmation at least 4 weeks after the initial evaluation.

The primary endpoint was the MTD of anlotinib, at which less than $33 \%$ of patients experienced a DLT in the first treatment cycle. A DLT involving hematological toxicity was defined as grade 4 and above, non-hematological toxicity as grade 3 and above, and liver and kidney function injury as grade 2 and above. The secondary endpoints included PFS and OS. PFS was defined as the time from the date of randomization to the date of disease progression or death. OS was defined as the time from the date of randomization to the date of death.

\section{Statistical analysis}

The recruitment of a minimum of 3 patients and a maximum of 18 patients was planned in accordance with the $3+3$ study design. Analyses were based on an Apr 10, 2020, database lock. All patients who received at least one dose of the investigational drug were included in the safety

Table 2 Overview of adverse events and discontinuations

\begin{tabular}{ll}
\hline $\mathrm{n}(\%)$ & Anlotinib $(\mathrm{n}=8)$ \\
\hline Total patients with $\geq 1 \mathrm{AE}$ & $8(100.0)$ \\
Serious AE & $3(37.5)$ \\
Severe AE (grade $\geq 3)$ & $5(62.5)$ \\
AE leading to death & $0(0.0)$ \\
AE leading to withdrawal from treatment & $2(25.0)$ \\
AE leading to dose modification/interruption & $5(62.5)$ \\
Treatment-related AE & $8(100.0)$ \\
Treatment-related serious AE & $3(37.5)$ \\
\hline
\end{tabular}


assessment, and those who completed at least one cycle of the treatment were eligible for efficacy evaluation. The baseline demographic characteristics and frequency of adverse events are summarized with descriptive statistics. Two-sided 95\% exact CIs were calculated for the ORR and DCR using the Clopper-Pearson method, and estimated time-to-event endpoints were calculated with the Kaplan-Meier method with two-sided 95\% CIs for medians. All statistical analyses were carried out with SAS 9.1.3 software.

\section{Results}

\section{Demographic characteristics}

A total of 8 patients were enrolled in this trial between April 2019 and November 2019. All patients were untreated before enrollment and had histologically confirmed adenocarcinoma without sensitizing EGFR/ALK/ROS1 alterations (Table 1). The median age was 62 years (39.0-69.0), and 7 of them had an ECOG performance status of 1.

\section{DLT and MTD}

In the study, there were 4 patients in the $12 \mathrm{mg}$ dose group and 4 patients in the $10 \mathrm{mg}$ dose group. Two DLTs were observed in two pts separately at anlotinib $12 \mathrm{mg}$ (grade 3 hand-foot syndrome and grade 3 appetite loss). The patient with appetite loss stopped anlotinib; the patient with handfoot syndrome reduced their anlotinib dose. Another patient with grade 3 hypertension and grade 2 proteinuria resumed anlotinib after suspension.

No DLTs occurred at $10 \mathrm{mg}$. Only 1 patient treated with $10 \mathrm{mg}$ anlotinib experienced grade 3 TREA (thrombocytopenia); therefore, the MTD was determined to be $10 \mathrm{mg}$, at

Table 3 Summary of most common TRAEs reported in $\geq 20 \%$ of patients

\begin{tabular}{|c|c|c|c|c|c|c|}
\hline & & AE, n (\%) & & & & $\geq$ Grade III AE \\
\hline & $12 \mathrm{mg}(\mathrm{n}=4)$ & $10 \mathrm{mg}(\mathrm{n}=4)$ & Total & $12 \mathrm{mg}(\mathrm{n}=4)$ & $10 \mathrm{mg}(\mathrm{n}=4)$ & Total \\
\hline TRAEs & $4(100.0)$ & $4(100.0)$ & $8(100.0)$ & $4(100.0)$ & $1(25.0)$ & $5(62.5)$ \\
\hline Appetite down & $4(100.0)$ & $3(75.0)$ & $7(87.5)$ & $0(0.0)$ & $0(0.0)$ & $2(25.0)$ \\
\hline Hypertriglyceridemia & $2(50.0)$ & $3(75.0)$ & $5(62.5)$ & $0(0.0)$ & $0(0.0)$ & $0(0.0)$ \\
\hline $\begin{array}{l}\text { Alanine transaminase } \\
\text { elevation }\end{array}$ & $3(75.0)$ & $2(50.0)$ & $5(62.5)$ & $0(0.0)$ & $0(0.0)$ & $0(0.0)$ \\
\hline $\begin{array}{l}\text { Aspartate transaminase } \\
\text { elevation }\end{array}$ & $2(50.0)$ & $3(75.0)$ & $5(62.5)$ & $0(0.0)$ & $0(0.0)$ & $0(0.0)$ \\
\hline Hypertension & $3(75.0)$ & $1(25.0)$ & $4(50.0)$ & $2(50.0)$ & $0(0.0)$ & $2(25.0)$ \\
\hline Thrombocytopenia & $2(50.0)$ & $2(50.0)$ & $4(50.0)$ & $0(0.0)$ & $1(25.0)$ & $1(12.5)$ \\
\hline Nausea & $1(25.0)$ & $3(75.0)$ & $4(50.0)$ & $0(0.0)$ & $0(0.0)$ & $0(0.0)$ \\
\hline Fatigue & $2(50.0)$ & $2(50.0)$ & $4(50.0)$ & $0(0.0)$ & $0(0.0)$ & $0(0.0)$ \\
\hline Neutropenia & $1(25.0)$ & $2(50.0)$ & $3(37.5)$ & $0(0.0)$ & $0(0.0)$ & $0(0.0)$ \\
\hline Hypercholesterolemia & $1(25.0)$ & $2(50.0)$ & $3(37.5)$ & $0(0.0)$ & $0(0.0)$ & $0(0.0)$ \\
\hline Leukopenia & $0(0.0)$ & $3(75.0)$ & $3(37.5)$ & $0(0.0)$ & $0(0.0)$ & $0(0.0)$ \\
\hline Dizziness & $1(25.0)$ & $2(50.0)$ & $3(37.5)$ & $0(0.0)$ & $0(0.0)$ & $0(0.0)$ \\
\hline Hand-foot syndrome & $2(50.0)$ & $0(0.0)$ & $2(25.0)$ & $1(25.0)$ & $0(0.0)$ & $1(12.5)$ \\
\hline Diarrhea & $2(50.0)$ & $0(0.0)$ & $2(25.0)$ & $1(25.0)$ & $0(0.0)$ & $1(12.5)$ \\
\hline Vomiting & $2(50.0)$ & $0(0.0)$ & $2(25.0)$ & $0(0.0)$ & $0(0.0)$ & $0(0.0)$ \\
\hline Gum pain & $1(25.0)$ & $1(25.0)$ & $2(25.0)$ & $0(0.0)$ & $0(0.0)$ & $0(0.0)$ \\
\hline Proteinuria & $1(25.0)$ & $1(25.0)$ & $2(25.0)$ & $0(0.0)$ & $0(0.0)$ & $0(0.0)$ \\
\hline Hypothyroidism & $1(25.0)$ & $1(25.0)$ & $2(25.0)$ & $0(0.0)$ & $0(0.0)$ & $0(0.0)$ \\
\hline Anemia & $0(0.0)$ & $2(50.0)$ & $2(25.0)$ & $0(0.0)$ & $0(0.0)$ & $0(0.0)$ \\
\hline Hoarseness & $1(25.0)$ & $1(25.0)$ & $2(25.0)$ & $0(0.0)$ & $0(0.0)$ & $0(0.0)$ \\
\hline $\begin{array}{l}\text { Thyroid stimulating } \\
\text { hormone elevation }\end{array}$ & $2(50.0)$ & $0(0.0)$ & $2(25.0)$ & $0(0.0)$ & $0(0.0)$ & $0(0.0)$ \\
\hline
\end{tabular}

$A E$ adverse event, TRAEs treatment-related adverse events 
Fig. 1 Best change from baseline in sum of longest target lesion diameter per patient

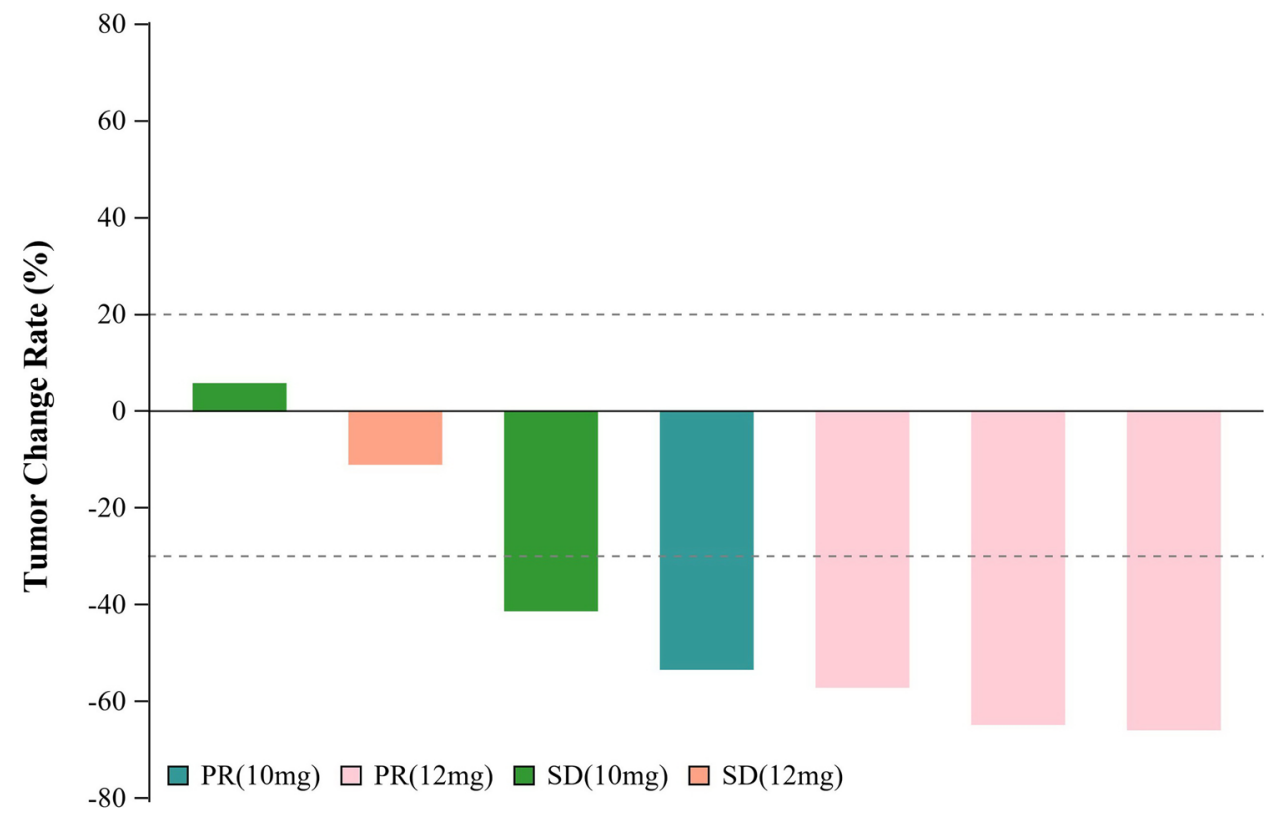

which less than $33 \%$ of pts experienced a DLT when treated with the anlotinib-platinum-pemetrexed combination.

\section{Safety}

AEs were monitored throughout the treatment until 30 days after termination of the trial. AEs occurred in all patients, and 5 of them experienced grade 3 AEs (Table 2). The most frequent AEs were appetite loss (87.5\%), alanine transaminase elevation (62.5\%), aspartate transaminase elevation (62.5\%), thrombocytopenia (50\%), nausea (50\%), hypertension (50\%) and fatigue (50\%). Grade 3 treatment-related adverse events (TRAEs) included appetite loss $(n=2)$, hypertension $(n=2)$, thrombocytopenia $(\mathrm{n}=1)$, diarrhea $(\mathrm{n}=1)$ and hand-foot syndrome $(n=1)$ (Table 3$)$. No grade 4 or grade 5 TRAEs were observed during the treatment.

\section{Efficacy}

The tumor response was assessed in 7 cases: $57.14 \%$ of cases had a confirmed objective response (95\% CI: $18.41 \%$, $90.10 \%)$ and $100 \%$ had confirmed disease control (95\% CI:
Fig. 2 The change curve with time in sum of longest target lesion diameter per patient

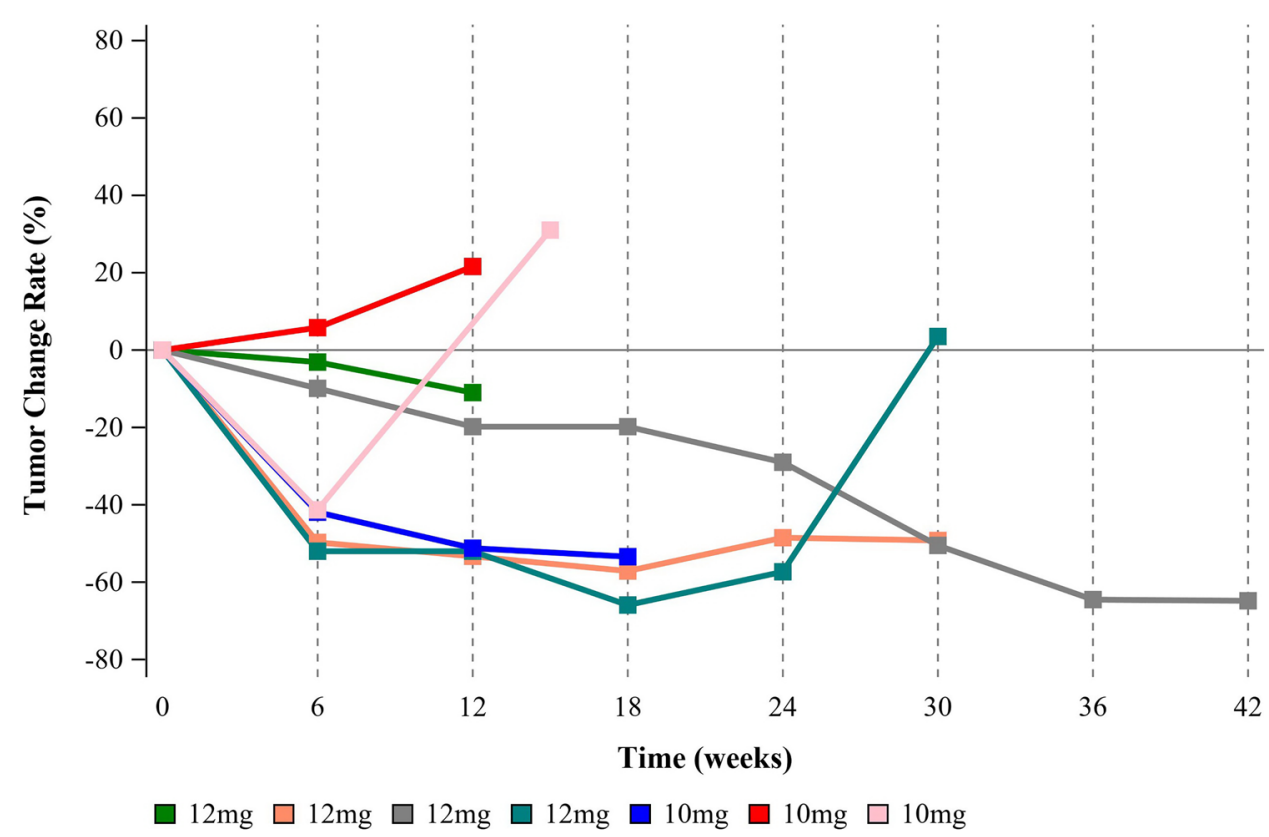


$59.04 \%, 100.00 \%)$. One patient withdrew consent before completing the first cycle. Among the evaluable cases, 6 exhibited tumor shrinkage (Fig. 1). Four (57.14\%) had a confirmed PR, 3 (42.86\%) had SD, and no PD was reported. At the data cutoff on Apr 10, 2020, six pts had terminated the treatment, predominantly due to disease progression. With a median follow-up of 10.05 months $(95 \% \mathrm{CI}: 5.59,11.40)$, the median PFS time was 7.00 months (95\% CI: 2.76 to NE).

Of the four responders, three were in the $12 \mathrm{mg}$ group, and another was in the $10 \mathrm{mg}$ group (Fig. 1). The responder who experienced the maximum tumor shrinkage was in the high-dose group with a response by the first scan (Fig. 2). At the time of the analysis, one subject in the $12 \mathrm{mg}$ group was still in this trial and showed sustained remission after 4 cycles of the combination treatment and 10 cycles of anlotinib maintenance (Fig. 2).

\section{Discussions}

The tolerability, safety and efficacy of anlotinib combined with chemotherapy were explored in this study, and it was demonstrated that the anlotinib-platinum-pemetrexed regimen was well tolerated in advanced NSCLC patients at an anlotinib dose of $10 \mathrm{mg}$. Moreover, the combination therapy demonstrated promising antitumor activity in these patients with an acceptable safety profile.

Overall, AEs in our study were mild, and the majority were grade 1 or 2 . Specific adverse events related to anlotinib, such as hypertension and hand-foot syndrome, were in the range of incidence reported in previous trials involving anlotinib [10]. On the other hand, the combination therapy resulted in a higher occurrence of hematological toxicities such as neutropenia, thrombocytopenia, leukopenia and anemia than that in response to platinum-pemetrexed alone, indicating an additive myelosuppressive effect of anlotinib, which has been mentioned by other investigators [11]. However, the hematological events significantly declined in the $10 \mathrm{mg}$ group, and the majority of these events were fully resolved. Overall, there was no evidence that anlotinib potentiated chemotherapy toxicity, and each individual drug could be administered in combination at the recommended dosage.

Remarkably, the tumor response of anlotinib-platinumpemetrexed in this trial $(57.14 \%)$ was encouraging, since pemetrexed-cisplatin alone only showed a response rate of $30.6 \%$ in the JMDB study, indicating a synergistic effect of anlotinib. Furthermore, we reported a median PFS of 7.00 months, and to the best of our knowledge, this is the first reported PFS of the APP regimen for treatment-naïve NSCLC. These favorable results were mainly attributed to the high selectivity and potent inhibition of VEGFR2 as well as broad inhibitory effects of anlotinib on other proangiogenic pathways $[12,13]$. In addition to its antiangiogenic activity, anlotinib suppresses tumor cell growth and migration by blocking c-Kit, c-Met and RET [14]. Collectively, our findings suggested that the combined treatment was efficacious and could be an alternative option for nonsquamous NSCLC.

Limitations to the findings included the small size of the cohort, which makes it difficult to draw firm conclusions on the antitumor efficacy of this regimen. In addition, the single-arm design of the trial limited its ability to directly compare results with those in response to chemotherapy alone. Considering these findings, the APP regimen could be further validated by controlled trials in the future.

Acknowledgements Not applicable.

Authors' contributions M.J.H and Y.L were responsible for the conception and design of the study. M.J.H, Y.M.L, M.Y were responsible for acquisition and analysis of data; furthermore, Y.Y.L conducted statistical analysis. M.J.H drafted the manuscript; Y.L revised and commented the draft, and all authors read and approved the final version of the manuscript.

Funding Not applicable.

Data availability The datasets used or analyzed during the current study are available from the corresponding author on reasonable request.

\section{Declarations}

Ethics approval and consent to participate This study was approved by the Ethics Committee of West Hospital of China, Sichuan University. All subjects agree to participate.

Consent for publication Written informed consent for publication was obtained from all participants.

Informed consent Written informed consent was obtained from all the subjects.

Research involving human participants and/or animals The study was performed in accordance with the ethical standards as laid down in the 1964 Declaration of Helsinki and its later amendments or comparable ethical standards.

Conflict of interests The authors declare that there was no competing interest in the study. The authors declare there is no conflict of interests.

Open Access This article is licensed under a Creative Commons Attribution 4.0 International License, which permits use, sharing, adaptation, distribution and reproduction in any medium or format, as long as you give appropriate credit to the original author(s) and the source, provide a link to the Creative Commons licence, and indicate if changes were made. The images or other third party material in this article are included in the article's Creative Commons licence, unless indicated otherwise in a credit line to the material. If material is not included in the article's Creative Commons licence and your intended use is not permitted by statutory regulation or exceeds the permitted use, you will 
need to obtain permission directly from the copyright holder. To view a copy of this licence, visit http://creativecommons.org/licenses/by/4.0/.

\section{References}

1. Laurie SA, Solomon BJ, Seymour L, Ellis PM, Goss GD, Shepherd FA, Boyer MJ, Arnold AM, Clingan P, Laberge F, Fenton D, Hirsh V, Zukin M, Stockler MR, Lee CW, Chen EX, Montenegro A, Ding K, Bradbury PA (2014) Randomised, double-blind trial of carboplatin and paclitaxel with daily oral cediranib or placebo in patients with advanced non-small cell lung cancer: NCIC Clinical Trials Group study BR29. Eur J Cancer 50:706-712

2. Paz-Ares LG, Biesma B, Heigener D, von Pawel J, Eisen T, Bennouna J, Zhang L, Liao M, Sun Y, Gans S, Syrigos K, Le Marie E, Gottfried M, Vansteenkiste J, Alberola V, Strauss UP, Montegriffo E, Ong TJ, Santoro A and Group NREUSIS (2012) Phase III, randomized, double-blind, placebo-controlled trial of gemcitabine/cisplatin alone or with sorafenib for the first-line treatment of advanced, nonsquamous non-small-cell lung cancer. J Clin Oncol 30:3084-3092

3. Crino L, Dansin E, Garrido P, Griesinger F, Laskin J, Pavlakis N, Stroiakovski D, Thatcher N, Tsai CM, Wu YL, Zhou C (2010) Safety and efficacy of first-line bevacizumab-based therapy in advanced non-squamous non-small-cell lung cancer (SAiL, MO19390): a phase 4 study. Lancet Oncol 11:733-740

4. Jardim DL, Gagliato Dde M, Ribeiro KB, Shimada AK, Katz A (2012) Bevacizumab as first-line therapy in advanced nonsmall-cell lung cancer: a brazilian center experience. Drugs R D 12:207-216

5. Zhou C, Wu YL, Chen G, Liu X, Zhu Y, Lu S, Feng J, He J, Han B, Wang J, Jiang G, Hu C, Zhang H, Cheng G, Song X, Lu Y, Pan H, Zheng W, Yin AY (2015) BEYOND: A Randomized, Double-Blind, Placebo-Controlled, Multicenter, Phase III Study of First-Line Carboplatin/Paclitaxel Plus Bevacizumab or Placebo in Chinese Patients With Advanced or Recurrent Nonsquamous Non-Small-Cell Lung Cancer. J Clin Oncol 33:2197-2204
6. Qin S, Li A, Yi M, Yu S, Zhang M, Wu K (2019) Recent advances on anti-angiogenesis receptor tyrosine kinase inhibitors in cancer therapy. J Hematol Oncol 12:27

7. Han B, Li K, Wang Q, Zhang L, Shi J, Wang Z, Cheng Y, He J, Shi Y, Zhao Y, Yu H, Zhao Y, Chen W, Luo Y, Wu L, Wang X, Pirker R, Nan K, Jin F, Dong J, Li B, Sun Y (2018) Effect of Anlotinib as a Third-Line or Further Treatment on Overall Survival of Patients With Advanced Non-Small Cell Lung Cancer: The ALTER 0303 Phase 3 Randomized Clinical Trial. JAMA Oncol 4:1569-1575

8. Zhou M, Chen X, Zhang H, Xia L, Tong X, Zou L, Hao R, Pan J, Zhao X, Chen D, Song Y, Qi Y, Tang L, Liu Z, Gao R, Shi Y, Yang Z (2019) China National Medical Products Administration approval summary: anlotinib for the treatment of advanced nonsmall cell lung cancer after two lines of chemotherapy. Cancer Commun (Lond) 39:36

9. Syed YY (2018) Correction to: Anlotinib: First Global Approval. Drugs 78:1287

10. Califano R, Tariq N, Compton S, Fitzgerald DA, Harwood CA, Lal R, Lester J, McPhelim J, Mulatero C, Subramanian S, Thomas A, Thatcher N, Nicolson M (2015) Expert Consensus on the Management of Adverse Events from EGFR Tyrosine Kinase Inhibitors in the UK. Drugs 75:1335-1348

11. Jain RK (2005) Antiangiogenic therapy for cancer: current and emerging concepts. Oncology (Williston Park) 19:7-16

12. Lin B, Song X, Yang D, Bai D, Yao Y, Lu N (2018) Anlotinib inhibits angiogenesis via suppressing the activation of VEGFR2, PDGFRbeta and FGFR1. Gene 654:77-86

13. Xie C, Wan X, Quan H, Zheng M, Fu L, Li Y, Lou L (2018) Preclinical characterization of anlotinib, a highly potent and selective vascular endothelial growth factor receptor-2 inhibitor. Cancer Sci 109:1207-1219

14. Shen G, Zheng F, Ren D, Du F, Dong Q, Wang Z, Zhao F, Ahmad R, Zhao J (2018) Anlotinib: a novel multi-targeting tyrosine kinase inhibitor in clinical development. J Hematol Oncol 11:120

Publisher's Note Springer Nature remains neutral with regard to jurisdictional claims in published maps and institutional affiliations. 